\title{
SOME INEQUALITIES FOR STOP-LOSS PREMIUMS
}

\author{
H. Bühlmans, B. Gagliardi, H. U. Gerber, E. Straub \\ Zürich and Ann Arbor
}

\section{A certain family of premium calculation principles}

In this paper any given risk $S$ (a random variable) is assumed to have $a$ (finite or infinite) mean. We enforce this by imposing $E\left[S^{-}\right]<\infty$.

Let then $v(t)$ be a twice differentiable function with

$$
v^{\prime}(t)>0, v^{\prime \prime}(t) \geq 0,-\infty<t<+\infty
$$

and let $z$ be a constant with $0 \leq z \leq \mathrm{I}$.

We define the premium $P$ as follows

$$
P=\sup \{Q \mid-\infty<Q<+\infty, E[v(S-z Q)]>v((\mathrm{I}-z) Q)\}
$$

or equivalently

$P=\sup \left\{Q \mid-\infty<Q<+\infty, v^{-1} o E[v(S-z Q)]>(\mathrm{I}-z) Q\right\}$.

Notation: $v^{-1}(\infty)=\infty$.

The definitions ( $I$ ) and (equivalently) (2) are meaningful because of the

Lemma: a) $E[v(S-z Q)]$ exists for all $Q \in(-\infty,+\infty)$.

b) The set $\{Q \mid-\infty<Q<+\infty, E[v(S-z Q)]>v((1-z) Q)\}$ is not empty.

Proof: a) $E\left[v^{-}(S-z Q)\right] \leq v^{-}(0) \cdot P[S \geq z Q]+v^{\prime}(0) \int_{S<x Q}(z Q-S) d P(S)$

$$
\leq v^{-}(0) \cdot P[S \geq z Q]+v^{\prime}(0)\left[z Q+E\left(S^{-}\right)\right]<\infty
$$

b) Because of a) $E[v(S-z Q)]$ is al waysfinite or equal to $+\infty$ If $v(-\infty)=-\infty$ then $E[v(S-z Q)]>v((\mathrm{I}-z) Q)$ is satisfied for sufficiently small $Q$. The left hand side of the inequality is a nonincreasing continuous function in $P$ (strictly decreasing if $z>0$ ), while the right hand 
side is a nondecreasing continuous function in $Q$ (strictly increasing if $z<\mathrm{I}$ ).

If $v(-\infty)=c$ finite then $E[v(S-z Q)]>c$

(otherwise $S$ would need to be equal to $-\infty$ with probability I) and again $E[v(S-z Q)]>v((I-z) Q)$ is satisfied for sufficiently small $Q$.

From the lemma we conclude the following useful

Corrolary: There are two cases to be distinguished

a) finite case: There exists $Q^{*}$ (finite) with

$$
E\left[v\left(S-z Q^{*}\right)\right]=v\left((\mathrm{I}-z) Q^{*}\right)
$$

or equivalently

$$
v^{-1} O E\left[v\left(S-z Q^{*}\right)\right]=(I-z) Q^{*}
$$

then $P=Q^{*}$.

b) infinite case: Otherwise $P=+\infty$.

Proof: From the proof of the lemma it is obvious that $Q^{*}$ under a) coincides with the supremum defining $P$.

Our premium calculation principle is determined by the choice of the function $v$ and the constant $z$ satisfying the above conditions. It satisfies the following very desirable postulates: For any risk $S$, for which the premium $P$ exists,

$$
\begin{aligned}
& P_{1}: P \geq E[S] \\
& P_{2}: P \leq \operatorname{Max}[S]
\end{aligned}
$$

Here Max [S] denotes the right hand end point of the range of $S$. Proof: For $P_{1}$ we start with equation (2) and make use of Jensen's inequality: $P$ is the least upper bound of the set of $Q$ 's for which

$$
(I-z) Q<v^{-1} \mathrm{O} E[v(S-z Q)] .
$$

By Jensen's inequality

$$
v^{-1} O E[v(S-z Q)] \geq v^{-1} \circ v(E[S-z Q])=E[S]-z Q .
$$


The set of $Q$ 's for which

$Q<E[S]$ is hence a subset and its supremum

$E[S]$ can not exceed the supremum $P$ of the bigger set.

For $P_{2}$ we start with equation $\left(2^{*}\right)$ (only the case $\operatorname{Max}[S]<\infty$ needs to be proved) and get

$$
\begin{aligned}
& (\mathrm{I}-z) P=v^{-1} \circ E[v(S-z P)] \\
& \leq v^{-1} \circ \operatorname{Max}[v(S-z P)] \\
& =v^{-1} \circ v(\operatorname{Max}[S-z P]) \\
& =\operatorname{Max}[S]-z P
\end{aligned}
$$

Remarks:

I) If $z=\mathrm{I}$, we obtain the principle of zero utility,

$$
P=\sup \{Q \mid E[u(Q-S)]<u(0)\}
$$

by setting $u(t)=-v(-t)$.

2) If $z=0$, wc obtain the mean value principle,

$$
P=v^{-1} \circ E[v(S)] \text {. }
$$

3) In the case where the function $v$ is linear or exponential, the premium calculation principle dues not depend on the value of $z$.

\section{Partial Ordering among risks}

I.et $G(x), H(x)$ be any distributions on the real line. Then we say that $G<H$, if

$$
\text { (PO) } \int_{1}^{\infty}(x-t) d G(x) \leq \int_{1}^{\infty}(x-t) d H(x),-\infty<t<\infty .
$$

Condition (b) simply means that for any retention limit $t$ the net stoploss premium for a risk whose cdf is $G$ is not higher than the one for a risk whose cdf is $H$. We do allow the case where the integrals become infinite. Integration by parts leads to the following equivalent condition:

$$
\left(P O^{\prime}\right) \int_{i}^{\infty}[I-G(x)] d x \leq \int_{i}^{\infty}[\mathrm{I}-H(x)] d x .
$$

The equivalence of $(P O)$ and $\left(P O^{\prime}\right)$ in the case of infinite integrals is e.g. proved in Feller II, page I 50. 
Let us now consider two stop-loss arrangements based on risks with $\operatorname{cdf} G$ and $H$, respectively. Let $P_{\alpha}^{G}, P_{\alpha}^{H}$ denote the corresponding stop-loss premiums ( $\alpha=$ retention limit). For example, $P_{\alpha}^{H}$ is obtained as the least upper bound of the set of $Q^{\prime}$ s for which

$$
v((I-z) Q)<v(-z Q) H(\alpha)+\int_{\alpha} v(t-\alpha-z Q) d H(t)
$$

and in the finite case as the unique solution of

$$
v\left[(\mathrm{I}-z) P_{\alpha}^{H}\right]=v\left(-z P_{\alpha}^{H}\right) H(\alpha)+\int_{a}^{\infty} v\left(t-\alpha-z P_{\alpha}^{H}\right) d H(t)
$$

The importance of the partial ordering introduced in this section becomes evident in the following theorem:

$$
\begin{aligned}
& \text { Theorem I: Suppose } G<H \\
& \text { Then } P_{\alpha}^{G} \leq P_{\alpha}^{H},-\infty<\alpha<+\infty
\end{aligned}
$$

Proof: If $P_{a}^{H}=\infty$ nothing is to be proved. We therefore assume $P_{a}^{H}$ finite which implies $\int_{1}^{\infty}[I-H(x)] d x<\infty$ for all $t \varepsilon(-\infty,+\infty)$.

If we integrate in equation $\left(3^{*}\right)$ twice by parts, we obtain:

$$
\begin{aligned}
v\left((\mathrm{I}-z) P_{\alpha}^{H}\right) & =v\left(-z P_{\alpha}^{H}\right)+\int_{\alpha}^{\infty} v^{\prime}\left(t-\alpha-z P_{\alpha}^{H}\right)[\mathrm{I}-H(t)] d t \\
& =v\left(-z P_{\alpha}^{H}\right)+v^{\prime}\left(-z P_{\alpha}^{H}\right) \int_{\alpha}^{\infty}[\mathrm{I}-H(t)] d t \\
& +\int_{\alpha}^{\infty} v^{\prime \prime}\left(t-\alpha-z P_{\alpha}^{H}\right) \int_{\alpha}^{\infty}[\mathrm{I}-H(x)] d x d t .
\end{aligned}
$$

Now we estimate the last two terms from below, replacing $H$ by $G$ and using condition $\left(P O^{\prime}\right)$. By reversing the last step (integration by parts) we arrive at

$$
v\left[(\mathrm{I}-z) P_{\alpha}^{H}\right] \geq v\left(-z P_{\alpha}^{H}\right)+\dot{\int}_{\alpha} v^{\prime}\left(t-\alpha-z P_{\alpha}^{H}\right)[\mathrm{I}-G(t)] d t
$$

and therefore $P_{\alpha}^{G} \leq P_{\alpha}^{H}$

q.e.d.

We postpone examples to sections 3 and 4 and conclude this section with some useful lemmas. Their content is essentially that 
the partial ordering is preserved under mixing and under convolution.

Lemma $I$ : Let $\left(G_{n}\right),\left(H_{n}\right)$ be sequences of distributions, and let $\left(p_{n}\right)$ be a discrete probability distribution. If $G_{n}<H_{n}$ for all $n$, then

$$
\sum_{n} p_{n} G_{n}<\Sigma p_{n} H_{n}
$$

Proof: Apply monotone convergence theorem

Lemma 2: If $G<H$, then

$$
G * F<H * F
$$

Proof: To establish the validity of condition $\left(P O^{\prime}\right)$, we observe that

$$
\begin{aligned}
& \int_{1}^{\infty}[\mathrm{I}-G * F(x)] d x \\
& =\int_{1}^{\infty} \int_{-\infty}^{\infty}[\mathrm{I}-G(x-s)] d F(s) d x
\end{aligned}
$$

and by Fubini's theorem

$$
=\int_{-\infty}^{\infty} \int_{i=}^{\infty}[\mathrm{I}-G(y)] d y d F(s)
$$

The last expression shows that we obtain an upper bound if we replace $G$ by $H$.

q.e.d.

$$
\begin{aligned}
& \text { Lemma } 3: \text { If } G_{i}<H_{i},(i=\mathrm{I}, 2, \ldots, n) \text {, then } \\
& \qquad G_{1} * G_{2} * \ldots * G_{n}<H_{1} * H_{2} * \ldots * H_{n}
\end{aligned}
$$

Proof: Repeated application of Lemma 2 leads to

$$
\begin{aligned}
& G_{1} * G_{2} * G_{3} * \ldots * G_{n} \\
< & H_{1} * G_{2} * G_{3} * \ldots * G_{n} \\
< & H_{1} * H_{2} * G_{3} * \ldots * G_{n} \\
< & H_{1} * H_{2} * H_{3} * \ldots * G_{n} \quad \text { etc. }
\end{aligned}
$$




\section{Application I: Dangerous Distributions}

Definition: A distribution $H$ is called more dangerous than a distribution $G$ if $(A)$ the first moments say $\mu_{G}, \mu_{H}$ exist and $\mu_{G} \leq \mu_{H}$ and if $(B)$ there is a constant $\beta$ such that

$$
\begin{aligned}
& G(x) \leq H(x) \text { for } x<\beta \\
& G(x) \geq H(x) \text { for } x \geq \beta .
\end{aligned}
$$

Example $I$ : Let $G$ be unimodal with $G(a-)=0, G(b)=I$ for $-\infty<a<b<\infty$. Let $c, d$ be numbers such that $c \leq a, b \leq d$ and $(c+d) / 2 \geq \mu_{G}$. Then the uniform distribution over the interval $(c, d)$ is more dangerous than $G$.

Example 2: Let $F$ be a distribution with $F(a-)=0, F(b)=\mathrm{I}$ for $-\infty<a<b<\infty$. I.et

$$
G(x)=\left\{\begin{array}{l}
o \text { for } x<\mu_{F} \\
I \text { for } x \geq \mu_{F}
\end{array}\right.
$$

and

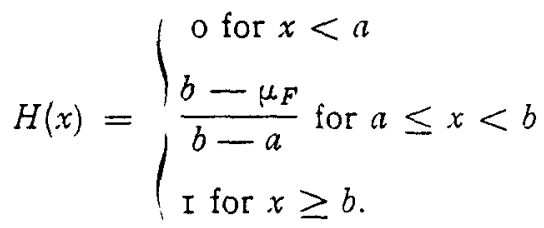

Then $F$ is more dangerous than $G$, and $H$ is more dangerous than $F$.

Theorem 2 : If $H$ is more dangerous than $G$, then $G<H$.

Proof: Condition $\left(P O^{\prime}\right)$ is obviously satisfied if $t \geq \beta$. If $t<\beta$. its validity can be seen as follows:

$$
\begin{aligned}
& \int_{1}^{\infty}[\mathrm{I}-G(x)] d x-\int_{1}^{\infty}[\mathrm{I}-H(x)] d x \\
& =\int_{1}^{\infty}[H(x)-G(x)] d x \\
& \leq \int_{-\infty}^{\infty}[H(x)-G(x)] d x=\mu_{G}-\mu_{H} \leq \text { o. } \quad \text { q.e.d. }
\end{aligned}
$$


Illustration $I$ : Let $S=S_{1}+S_{2}+\ldots+S_{n}$ be a sum of $n$ independent risks. If we replace each of these risks by a more dangerous risk, the stop-loss premium for the sum of these new risks will be at least as high as the stop-loss premium for $S$ (use Theorems I, 2 and Lemma 3).

Illustration 2: Let $S$ be a risk with a compound Poisson distribution, say with Poisson parameter $\lambda$ and amount distribution $F(x)$. We assume that $F(0)=0$ (only positive claims) and that $F(M)=\mathrm{I}$ for some $M>0$ (a claim amount is at most $M$ ), and let $\mu$ denote the mean of $F$ (i.e. the average claim amount). We compare $S$ with the two compound Poisson risks $S^{\mu}, S^{M}$ with fixed claim amounts $\mu, M$, respectively, and Poisson parameters $\lambda_{j} \Lambda=\lambda(\mu / L /)$, respectively. (Observe that $E\left(S^{\mu}\right)=E(S)=E\left(S^{M}\right)$.) From Example 2 (with $a=0, b=M$ ), Lemmas I, 3, and Theorems I, 2 we obtain inequalities for the corresponding stop-loss premiums:

$$
P_{\mathrm{x}}^{\prime \prime} \leq P_{\mathrm{x}} \leq P_{\mathrm{x}}^{M I} \text {. }
$$

In the case of net stop-loss premiums the second inequality has been proved by Gagliardi and Straub (Mitteilungen Vereinigung schweizerischer Versicherungsmathematiker, I974, Heft 2).

4. Application 2: Random sums of positive rishs

In this section we shall compare a distribution of the form

$$
G=(\mathrm{I}-q) F^{* 0} \perp q F, 0 \leq q \leq \mathrm{I}
$$

with one of the more general form

$$
H=\sum_{n=0}^{\infty} p_{n} F^{* n}
$$

where

$$
0 \leq p_{n} \leq \mathrm{I}, \sum_{n=1}^{\infty} p_{n}=\mathrm{I}
$$

Theorem 3: Suppose $F(0)=0$

If $\Sigma^{\infty} n p_{n}=q$, then $G<H$, where $G, H$ are given by (4), (5). 
Proof: Firstly, we show that

$$
F<\frac{n-I}{n} F^{* 0}+\frac{I}{n} F^{* n}, n=\mathrm{I}, 2, \ldots
$$

which is a special case of Theorem 3 .

To show the validity of condition $(P O)$ we introduce the independent random variables $X_{1}, X_{2}, \ldots X_{n}$ with common distribution $F$. Then condition $(P O)$ is equivalent to

$$
\sum_{i=1}^{n} E\left[\left(X_{i}-t\right)_{+}\right] \leq(n-\mathrm{I})(-t)_{+}+E\left[\left(\sum_{i=1}^{n} X_{i}-t\right)_{+}\right]
$$

But the corresponding inequality is satisfied for any outcomes of $X_{1}, X_{2}, \ldots, X_{n}$.

Secondly, we show that $G<H$ in the general case. Since

$$
\begin{aligned}
& H=\sum_{n=1}^{\infty} n p_{n}\left[\frac{n-I}{n} F^{* 0}+\frac{I}{n} F^{* n}\right]+(I-q) F^{* 0} \\
& G=\sum_{n=1}^{\infty} n p_{n} F+(I-q) F^{* 0}
\end{aligned}
$$

this follows from equation (6) and Lemma $x$.

Illustration: Individual versus collective model: The individual model is described by $n$ numbers $q_{i}, 0<q_{i} \leq \mathrm{I}$, and $n$ distributions $F_{i}$ with $F_{i}(0)=0$. We have in mind a portfolio consisting of $n$ components. Then $q_{t}$ is the probability that a claim occurs in component $i$, and $F_{i}$ is the distribution of its amount. I.et

$$
S^{\text {ind }}=S_{1}+S_{2}+\ldots+S_{n}
$$

denote the total claims of the portfolio, where

$$
\begin{aligned}
& \operatorname{Prob}\left(S_{i}=0\right)=\mathrm{I}-q_{i} \\
& \operatorname{Prob}\left(S_{i} \leq x\right)=\mathrm{I}-q_{i}+q_{i} F_{i}(x), x>0
\end{aligned}
$$

for $i=\mathrm{I}, 2, \ldots, n$. We assume that $S_{i}, S_{2}, \ldots, S_{n}$ are independent and denote the stop-loss premium for $S^{\text {ind }}$ by $P_{\alpha}^{\text {ind }}(\alpha=$ retention limit). 
A collective model is assigned to the individual model in a well known fashion: Let $S$ coll denote the compound Poisson random variable with

$$
\begin{aligned}
& \text { Poisson parameter } \quad \lambda=\sum_{i=1}^{n} q_{i} \\
& \text { Amount distribution } F=\sum_{i=1}^{n} q_{i} / \lambda F_{i} .
\end{aligned}
$$

Let $P_{\alpha}^{\text {coll }}$ denote the stop-loss premium for $S^{\text {coll }}$. By applying Theorem 3 to each of the $n$ components (replacing $S_{i}$ by a compound Poisson random variable with Poisson parameter $q_{i}$ and amount distribution $F_{i}$ ), we recognize from Theorem I and Lemma 3 that $P_{\alpha}^{\text {ind }} \leq P_{\alpha}^{\text {coll }}$. Thus a cautious reinsurer will piefer the collective model to the individual model. 